\title{
Methods for modeling the steering wheel torque of a steer-by-wire vehicle
}

\author{
Felix Heinrich ${ }^{1}$ (D) Jonas Kaste ${ }^{1} \cdot$ Sevsel Gamze Kabil ${ }^{1}$ - Michael Sanne ${ }^{1} \cdot$ Ferit Küçükay $^{2} \cdot$ Roman Henze $^{1,2}$. \\ Joachim Axmann ${ }^{1,2}$
}

Received: 14 June 2021 / Accepted: 25 October 2021 / Published online: 9 December 2021

(c) The Author(s) 2021

\begin{abstract}
Unlike electromechanical steering systems, steer-by-wire systems do not have a mechanical coupling between the wheels and the steering wheel. Therefore, a synthetic steering feel has to be generated to supply the driver with the necessary haptic information. In this paper, the authors analyze two approaches of creating a realistic steering feel. One is a modular approach that uses several measured and estimated input signals to model a steering wheel torque via mathematical functions. The other approach is based on an artificial neural network. It depends on steering and vehicle measurements. Both concepts are optimized and trained, respectively, to best fit a reference steering feel obtained from vehicle measurements. To carry out the analysis, the two approaches are evaluated using a simulation model consisting of a vehicle, a rack actuator, and a steering wheel actuator. The research shows that both concepts are able to adequately model a desired steering feel.
\end{abstract}

Keywords Steer-by-wire $\cdot$ Steering feel $\cdot$ Virtual development methods $\cdot$ Artificial neural networks $\cdot$ Clustering

\section{Introduction}

In recent years, a steadily increasing number of driver assistance systems have found their way into vehicle technology. Work on partially and fully autonomous driving functions for automobiles has made great strides, and is also a high priority in research and development. To meet the equally increased demand for comfort in this context, the development of steer-by-wire systems is also being driven forward. By separating the mechanical connection between the wheels and the steering wheel, this type of steering allows, among other advantages, the wheels to move independently of the steering wheel, thus decoupling the driver. Unlike electromechanical steering systems, which currently represent the state of the art, steer-by-wire requires feedback to be synthetically generated for the driver. Some scientific studies are already looking at different strategies for

Felix Heinrich

felix.heinrich@volkswagen.de

1 Volkswagen AG, Berliner Ring 2, 38440 Wolfsburg, Germany

2 Institut für Fahrzeugtechnik, Technische Universität Braunschweig, Hans-Sommer-Straße 4, 38106 Brunswick, Germany generating a steering feel $[1,6,8,10]$. However, the aim of the present investigations is to generate a steering feedback that corresponds to a brand-specific steering feel across different driving conditions. For this purpose, two different approaches are investigated simulatively and compared to a series production EPS system. One approach is to generate a steering wheel torque based on a simulated rack and pinion force. The second approach is to create the desired steering wheel torque using an artificial neural network. Both methods are based on recorded measurement data from a production vehicle, which is used to tune or train the algorithms. Another focus is on minimizing the training time of the neural network and improving the distribution of the training data sample using clustering.

In the following, first, the structure of the vehicle with its steer-by-wire system is described and the model is validated on the basis of measurement data. Subsequently, both approaches and the processes of optimization and training are described. A special attention is given to the clustering of the data to improve the training process. In the end, the results of each approach are shown and compared to one another. 


\section{System design}

The steer-by-wire system under consideration consists of two actuators: the steering rack actuator and the steering wheel actuator. The steering rack actuator controls a specific rack position according to the steering wheel position. It is connected to the steering wheel actuator via a redundant CAN bus connection. While the rack actuator realizes the commanded target position, the wheel actuator provides feedback to the driver. Both actuators each have two control units and have 6-phase windings of the electric machines, three of which are controlled by each control unit. This means that if one component fails, the system can continue to operate safely with half the torque.

The system is installed in a C-segment vehicle, which is represented by a simulation model in this study. The vehicle model is described in the following. Also, the models of the steering wheel and steering rack actuator as well as the simulation environment are described.

\subsection{Vehicle dynamics}

A linear bicycle model according to [17] is used to map the lateral dynamics of the real vehicle. Figure 1 depicts the forces and torques that determine the behavior.

The following differential equations describe the linear and angular momentum:

$m \cdot a_{y}=F_{y v}+F_{y h}$

$J_{z} \cdot \ddot{\psi}=F_{y v} \cdot l_{v}-F_{y h} \times l_{h}$.

The vehicle model's behavior has been adapted to the corresponding real vehicle by optimization. The single-track model is enhanced by including the body's roll behavior and the influences of roll and lateral force steering. To be able to describe dynamic effects in the lateral force build-up, a model according to Böhm is used [3]. It models the tire behavior and considers the relaxation length $l_{\mathrm{r}}$, the slip angle of the tire $\alpha$, and the slip stiffness $c_{\alpha}$ of the tire, and can be described by a first-order system

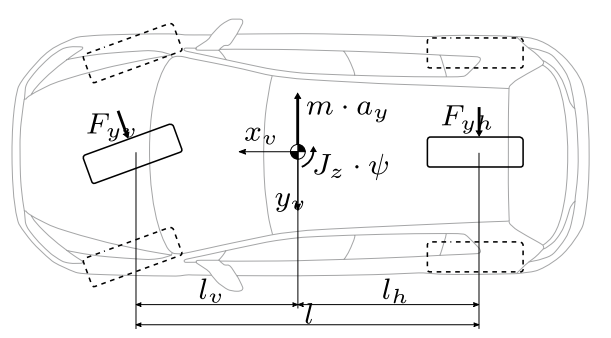

Fig. 1 Schematic representation of the underlying bicycle model
$F_{y}+\frac{l_{r}}{v} \cdot \frac{\mathrm{d} F_{y}}{\mathrm{~d} t}=c_{\alpha} \cdot \alpha$

With the help of these extensions, it is possible to reproduce lateral acceleration $a_{y}$, yaw rate $\psi$, and rack force $F_{\mathrm{ZS}}$ of a physical reference vehicle more precisely. The system is excited by the rack position, since it represents the interface between the vehicle and the steering system. The conversion of the rack position into a wheel angle is done via the kinematics of the chassis, taking into account the stiffness of wheel and steering bearings.

\subsection{Steering wheel actuator}

In addition to the input of the steering wheel angle by the driver, the steering wheel actuator is also responsible for generating the steering torque, thus giving the driver a feeling for the current driving situation. The steering wheel is connected to a permanently excited synchronous machine via a worm gear, similar to a column EPS. With a ratio of 18:1, the servo motor can generate up to $60 \mathrm{Nm}$ at the steering wheel. The torque applied to the steering wheel is measured by a torsion bar element between the steering wheel and gear stage. The system is modeled by a two-mass oscillator. The masses of the motor and the steering wheel, that are referenced by the indices $M_{1}$ and $S$, respectively, are connected by a spring with stiffness $c_{\mathrm{T}}$. Each mass has a rotational inertia $J$ and is acted upon by damping $d$ and a frictional force $F_{f}$. The systems can be described using the differential equations

$J_{\mathrm{M}_{1}} \ddot{\varphi}_{M_{1}}=c_{\mathrm{T}}\left(\varphi_{\mathrm{S}}-\varphi_{\mathrm{M}_{1}}\right)-d_{\mathrm{M}_{1}} \dot{\varphi}_{\mathrm{M}_{1}}-F_{\mathrm{f}, \mathrm{M}_{1}}$

$J_{\mathrm{S}} \ddot{\varphi}_{\mathrm{S}}=-c_{\mathrm{T}}\left(\varphi_{\mathrm{S}}-\varphi_{\mathrm{M}}\right)-d_{\mathrm{S}} \dot{\varphi}_{\mathrm{S}}-F_{\mathrm{f}, \mathrm{S}}$.

While a linear approach was chosen for damping, a more complex approach is required to account for friction. Friction modeling according to Stribeck has proven to be appropriate [23]. In addition to the mechanical properties of the actuator, a torque controller has also been designed, which is discussed in more detail in chapter 3 .

\subsection{Steering rack actuator}

To model the steering gear, its mechanical properties and the system's position controller are considered. Mechanically, the rack actuator can be described as an inertial mass with a frictional force acting upon it in addition to the chassis forces and the actuator torque. Thus, the following differential equation results for the description of the system behavior: 
$m_{\mathrm{T}} \cdot \ddot{x}_{\mathrm{R}}=M_{\mathrm{M}_{2}} \cdot i_{\mathrm{M}_{2}} \cdot r_{\mathrm{P}}-F_{\mathrm{f}, \mathrm{R}}-F_{\mathrm{R}}$.

Here, the mass $m_{\mathrm{T}}$ represents a virtual total mass which takes into account the actual rack mass and interprets the rotational inertia of the electric motor as a mass in rack level. $M_{\mathrm{M}_{2}}$ represents the motor torque which acts on the rack via the gear ratio $i_{\mathrm{M}_{2}}$ and the pinion radius $r_{\mathrm{P}}$. The frictional force of the gear rack is described by $F_{\mathrm{f}, \mathrm{R}}$. Like the steering wheel actuator's friction, it is modeled according to Stribeck. The force $F_{\mathrm{R}}$ is the rack force and represents the interface to the vehicle dynamics model in addition to the rack position $x_{\mathrm{R}}$. The position controller of the steering gear actuator is also included in the model. It is a cascaded controller that controls a target position as well as a target speed of the rack.

\subsection{Simulation environment}

The described components of the entire system were modeled in MATLAB/Simulink. A schematic representation of the modular structure of the model with the interfaces between the individual components is shown in Fig. 2.

Input variables are the steering wheel angle $\varphi_{\mathrm{S}}$, which is imposed by the driver, the steering angle speed $\dot{\varphi}_{\mathrm{S}}$, and the vehicle speed $v$. The steering wheel angle, minus the angle loss due to the stiffness of the torque sensor, is transferred as $\varphi_{\mathrm{S} \text {,red. }}$ to the steering gear actuator, which calculates and adjusts a target rack position from this based on the steering ratio. The actual rack position $x_{\mathrm{R}}$ is responsible for the lateral excitation of the vehicle dynamics model.

Via the chassis kinematics, this provides the rack force $F_{\mathrm{R}}$ based on the lateral forces of the front axle. Besides the internal friction and damping, it represents the counter force of the rack actuator. The estimated rack force $F_{\mathrm{R}, \text { est. }}$ is composed of the sum of the rack force $F_{\mathrm{R}}$ and the internal forces of the steering gear, and is fed into the steering wheel actuator. There, a target steering torque is generated on the basis of the input variables vehicle speed $v$, the estimated rack force $F_{\mathrm{R}, \text { est. }}$, steering wheel angle $\varphi_{\mathrm{S}}$, and its derivative $\dot{\varphi}_{\mathrm{S}}$, and is adjusted in the subsequent controller. As a result, the driver feels the steering wheel torque $M_{\mathrm{S}}$. A detailed description of the contents of torque generation and the controller can be found in the following chapter 3 .

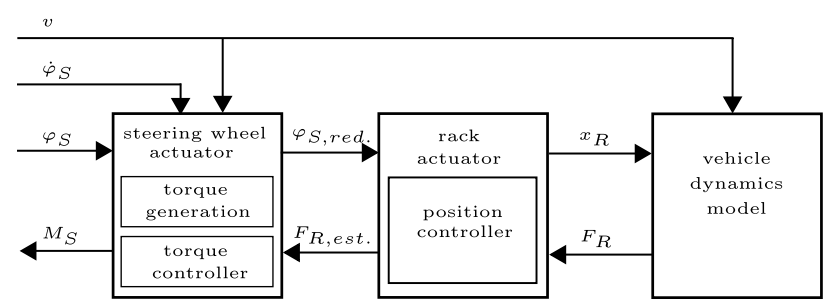

Fig. 2 Structure of the complete simulation model
The advantage of modeling three separate components is that each individual component, such as the steering wheel actuator, can be validated independently on the basis of their interfaces and exchanged as required. This means that not only different steering systems can be investigated but also the same steering systems can be simulated in different vehicles.

\subsection{Validation}

The three components of the simulation environment have been validated individually by comparing simulation results to measurement data. The vehicle dynamics model has proven to deviate less than $10 \%$ from actual test data in lateral acceleration $a_{y}$, yaw rate $\dot{\psi}$, and rack force $F_{\mathrm{R}}$ using sine steering maneuvers up to $2 \mathrm{~Hz}$ and a steering angle ramp with up to $85^{\circ} \mathrm{s}^{-1}$ reaching a lateral acceleration $a_{y}$ of $4 \mathrm{~m} \mathrm{~s}^{-1}$. Similar maneuvers have been used to validate the rack actuator model. It has proven to supply the necessary precision to represent the physical system. It is vital to the subject of this study; the feedback actuator model has been validated using a manual steering pattern. It includes slow transitions in direction as they unveil potential discrepancies in the actuators friction model. The upper graph in figure 3 depicts the manual maneuver's steering angle.

The simulated and the measured steering torque as well as the resulting simulation error are shown in the lower graph. According to [4], a range of $0.44-0.58 \mathrm{Nm}$ was identified as the perceptible tactile threshold. As the error is rarely higher than $0.2 \mathrm{Nm}$ and does not reach a higher maximum value than $0.5 \mathrm{Nm}$, the feedback actuator model is suitable for adequate representation of the physical actuator.
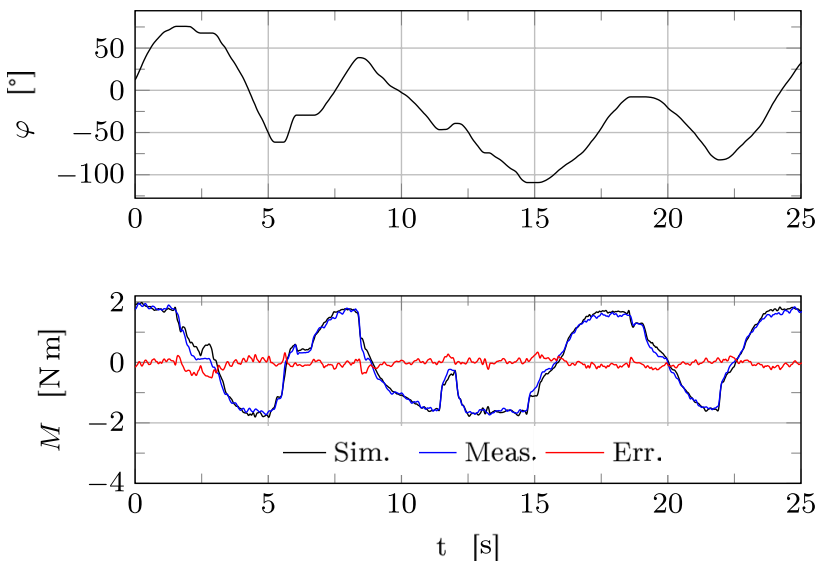

Fig. 3 Comparison of simulated and measured steering torque 


\section{Modular steering feel approach}

The aim of generating the steering feel is to reproduce a defined target steering feel as well as possible. In this study, the steering feel is intended to correspond to that of an electromechanical steering system. To define this steering feel, an EPS system was evaluated in road tests. Subsequently, the various components that make up the steering wheel torque were modeled. These were then optimized, so that the steering wheel torque generated corresponds to the reference steering wheel torque. The steering wheel torque is characterized by the reference torque generation and the torque controller, which are described in detail below.

\subsection{Target torque generation}

To simulate the steering torque, the torque-generating components of an EPS have been modeled similar to [6]. The components of this concept serve two purposes. They represents the steering functions of an EPS and the mechanical properties of such a system. The according structure is shown in Fig. 4.

The modular design has the advantage of being able to independently adjust the individual components by weighting factors and was derived from [6]. However, in this paper, the modules are combined differently and their content is adapted. The two torque-generating functions are an active return and the conversion of a calculated rack force $F_{\mathrm{R}}$ into a steering wheel torque hereafter named basic torque. The basic torque is generated from the calculated rack force $F_{\mathrm{R}}$ via a speed-dependent characteristic curve. Compared to an EPS system, it works like the inverse of a steering assistance function, where an assistance force is generated at the steering gear based on a measured torque. Here, a torque is generated based on the rack force $F_{\mathrm{R}}$. The components of the rack force are the estimated rack force $F_{\mathrm{R} \text {,est. }}$ and a synthetic friction $F_{\mathrm{F}}$ and damping force $F_{\mathrm{D}}$. The estimated rack

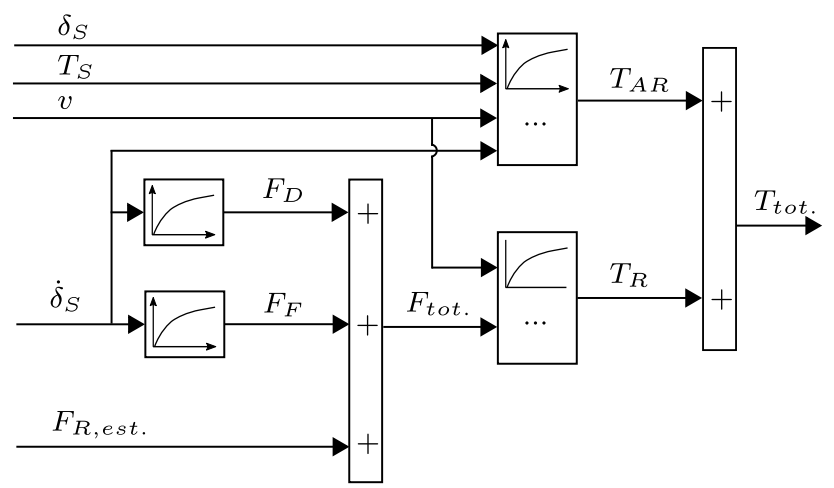

Fig. 4 Structure of the target torque generation derived from [6] force $F_{\mathrm{R} \text {,est. }}$ is computed based on the steering rack actuator's torque and its physical characteristics. Although a friction force is already included in the estimated rack force, the functions for friction and damping should be used to influence the steering feel independent of the mechanical friction of the steering system.

The active return module works like a cascaded control. A target steering wheel return velocity is calculated using characteristic curves from the steering wheel angle $\delta_{\mathrm{S}}$ taking into account the vehicle velocity $v$ and the measured steering wheel torque $T_{\mathrm{S}}$. This target return velocity is compared to the measured steering wheel speed. From the difference, again taking into account the vehicle velocity $v$ and the measured steering wheel torque $T_{\mathrm{S}}$, a return torque is formed which is added to the rack-force-based basic torque. The parameterization of the characteristic curves of active return and the module for calculating the base torque is derived from an EPS parameterization and adjusted by means of weighting factors to the forces $F_{\mathrm{D}}, F_{\mathrm{F}}$, the torques $T_{\mathrm{AR}}, T_{\mathrm{R}}$, as well as to the target return speed in the module of active return.

\subsection{Torque control}

The ability to represent a certain target behavior of the steering wheel torque with the given steer-by-wire system is significantly influenced by the stated properties of the steering wheel actuator. Inertia, damping and friction in the system generate torques that superimpose the actual target torque and have to be compensated. This is done via a controller. Due to its positive phase behavior and the simple implementation and tunability, a PD controller with feedforward control is used to control the steering wheel torque.

\subsection{Optimization}

The optimization of the steering feel is divided into two steps. First, the torque controller for the steering wheel torque was optimized. This is done based on the reaction of the simulation model of the steering wheel actuator to a target torque step. The criteria for this are based on [24], and include the response time of the controller and its maximum overshoot. After the controller has been optimized, the module for generating the target torque was optimized. This way, the controller behavior can be included in the optimization of the target torque and therefore be taken into account in the design of the target torque generation.

For the optimization of the target torque generation, the complete model consisting of vehicle dynamics, rack, and steering wheel actuator is used. The optimization is carried out on the basis of two driving maneuvers. Also, to reduce optimization time, for the scope of this study the tuning and optimization of the algorithm is limited to a constant 
velocity of $100 \mathrm{~km} / \mathrm{h}$. Using an evolutionary algorithm, the weighting factors of the target torque generation are modified in such a way that the simulated steering wheel torque at the torque sensor $T_{\mathrm{S}}$ corresponds as accurately as possible to the measured steering wheel torque from the real vehicle measurements $T_{\mathrm{S} \text {,mes. }}$. The quality of the simulated steering wheel torque $T_{\mathrm{S}}$ is represented by the root-mean-square error (RMSE)

$\mathrm{RMSE}=\sqrt{\frac{1}{n} \sum_{j=1}^{n}\left(T_{\mathrm{S}, \mathrm{mes} . j}-T_{\mathrm{S}, j}\right)^{2}}$.

\section{Model-free approaches for steering feel approximation}

As a model-free approach for mapping the steering feel in a steer-by-wire vehicle, a neural network is used in this paper. This approach was described in detail in [10,23]. Van Ende et al. examine the mapping of variable driving maneuvers between 0 and $100 \mathrm{~km} / \mathrm{h}$, and prove the basic suitability of the neural network to reproduce the desired target behavior. The selection of the training data set is done by a combination of data subsets, obtained from manual driving. For generalization of training performance, a statistical average of 20 networks with an identical topology is considered. For training [10] use representative, synthetic driving maneuvers that represent a wide range of the driving dynamic spectrum and are driven manually and repeated several times to provide variations within the training data.

In both studies, a subjective selection of different driving maneuvers is used to enable a holistic consideration of the vehicle dynamic potential of the investigated test vehicle and to provide an even distribution of relevant data points for the network training. To increase training performance, generalization properties and computing time for network training, within the present study, a clustering approach is integrated as an additional pre-processing step. The overall goal is to provide equally distributed information over a broad spectrum of different dynamic ranges for network training and at the same time to reduce the size of the training data set by removing less relevant or redundant data from the training data and integrating it into the test data set.

\subsection{Artificial neural networks}

Usually, with artificial neural networks (ANN) human actions, ways of working or thinking shall be transferred to machines, systems, and processes [2, 16, 18]. Depending on the area of application, more or less complex, abstracted models are available, which vary greatly in terms of the number of parameters and architecture depending on the complexity of the task to be modeled. Compared to the highly interconnected cell structure present in the human nervous system, however, the technical illustrations are strongly restricted due to limited resources in terms of computing and storage capacities. In [23], it is shown that neural networks with only one hidden layer and a few neurons are able to reproduce the nonlinear steering torque curve via vehicle dynamic inputs with high precision if training data are selected appropriately. In this study, feedforward networks are also considered, which is why the explanation of the theory is limited to this network architecture at this point. The essential elements of the artificial neural networks considered in this work are shown in Fig. 5.

At the top, an exemplary network with 4 inputs, 2 hidden layers with 10 neurons each and one output neuron is shown. At the bottom, the information processing steps can be seen for an exemplary neuron of the depicted network. The neural network consists of a layered structure of these idealized neurons. In the first layer, the inputs of the neurons represent the system inputs, which are composed of measurable variables that serve to describe the task to be modeled. In the following layers, the weighted outputs of upstream neurons serve as inputs for the neurons of the following layers. These are summed and processed by a predefined activation function to the neuron output. For a neuron $\mathrm{j}$ of the layer 1 , it follows:

$$
\begin{aligned}
& f_{a_{j}}^{(l)}=b_{j}^{(l)}+\sum_{i=1}^{q^{(l-1)}} o_{i}^{(l-1)} \cdot w_{i j}^{(l-1)} \\
& o_{j}^{(l)}=f_{o_{j}}^{(l)}\left(f_{a_{j}}^{(l)}\right) .
\end{aligned}
$$

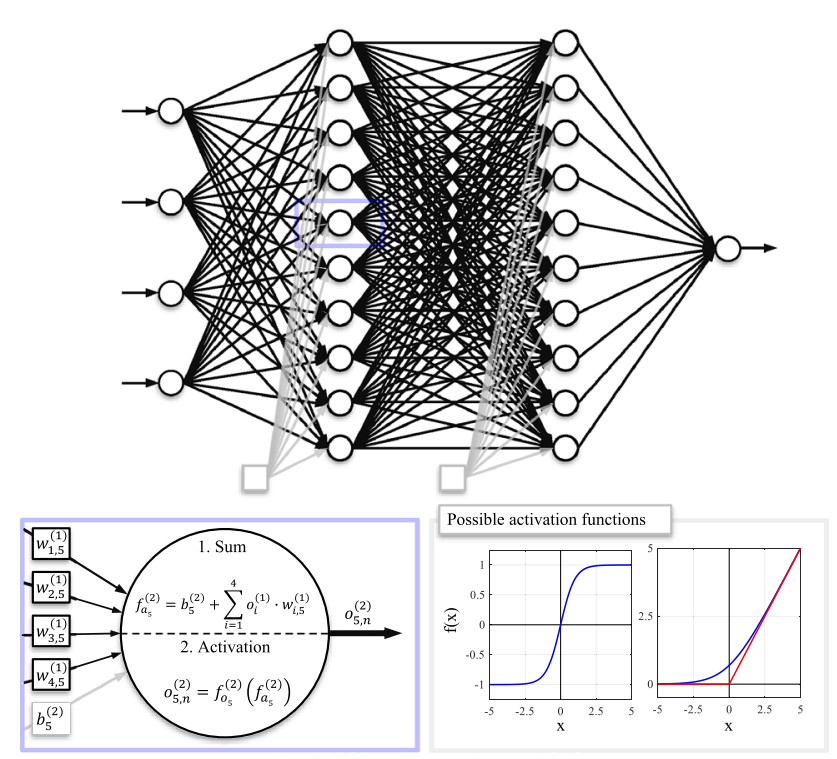

Fig. 5 Exemplary feedforward neural network, as well as an idealized neuron and typical activation functions 
The activation function $f_{o_{j}}^{(l)}$ is a design parameter that essentially determines the network output. Typical activation functions are shown in Fig. 5. The Tanh function represents a nonlinear, limited, sigmoid function which is used for a variety of applications in ANN [9, 19, 22, 23]. However, in recent years, unlimited functions, such as the ReLU or Softplus function, which represents a smooth approximation of the ReLU function, have gained increasing interest, since, in some cases, better training results have been achieved $[7,11$, 14].

The total network output for a network with two hidden layers, analogous to Fig. 5, can be calculated as follows:

$\mathbf{y}=\mathbf{o}^{(4)}=\mathbf{f}_{o}^{(4)}\left(\underline{\boldsymbol{W}}^{(3)} \cdot \mathbf{f}_{o}^{(3)}\left(\underline{\boldsymbol{W}}^{(2)} \cdot \mathbf{f}_{o}^{(2)}\left(\underline{\boldsymbol{W}}^{(1)} \cdot \mathbf{f}_{o}^{(1)}\left(\mathbf{i}^{(1)}\right)\right)\right)\right)$.

Here, $\mathbf{f}_{o}^{(1)}, \mathbf{f}_{o}^{(2)}, \mathbf{f}_{o}^{(3)}, \mathbf{f}_{o}^{(4)}$ correspond to the respective activation functions of the neurons of the four layers. The weight matrices between the individual layers are described with $\underline{W}^{(1)}, \underline{W}^{(2)}$, and $\underline{\boldsymbol{W}}^{(3)}$. The network input is marked $\mathbf{i}^{(1)}$. This information processing by the network is called forward propagation. To be able to model a system behavior with the help of the network, it is necessary to modify the weights in the network in the context of a training process in such a way that a suitable network output is calculated from a set of input data. This training process is usually performed by gradient descent-based training methods, which optimize the network weights epoch by epoch by backpropagating an error term. In the simplest case, the weight adjustment is done analogously to Eq. 11 with the vanilla update

$\Delta \underline{\boldsymbol{W}}=-\mu \cdot \nabla E(\underline{\boldsymbol{W}})$.

The weight change is done with the help of a pre-selected learning rate $\mu$ and the partial derivation of the error function on all weights in the network $\nabla E(\underline{W})$.

\subsection{Clustering to increase the efficiency of network training}

To ensure a training process for the neural network that is as efficient and robust as possible, a training data set is required that describes the later field of application as accurately as possible. For modeling steering torque, the relevant driving maneuvers from the lower to the highly dynamic range have to be represented by the training data. Effects such as changing tires and a variation of the vehicle behavior based on varying surfaces and lower force transmission, as well as different driving modes that potentially influence the desired steering torque are neglected in the present studies. The basic conditions for data set generation and model validation are described in the Sect. 4.3. Since not the entire available database, consisting of several million data points, is to be used, but the relevant maneuvers are to be isolated and redundant data points removed, an approach for the generation of subsets is examined in this paper. To achieve good generalization properties of the neural network and to increase training efficiency, the available data should be processed as equally distributed as possible according to driving dynamics and driving maneuvers. This is done by clustering with the k-means approach.

\subsubsection{K-means clustering}

The k-means algorithm, a partitioning clustering algorithm, is still a research topic $[5,13,21,25]$ and is often used to divide large amounts of data into smaller subsets [12, 20]. The data points to be clustered are represented by their mean values and automatically divided into $k$ clusters according to a specification or as described in [25]. The partitioning is based on the distances of single data objects to the centroids in multidimensional space. The centroids describe the center of a cluster. When clustering with the help of $k$-means, the following steps are performed iteratively until there are no changes in the assignment of the objects to individual clusters:

1. Initialization of $k$ clusters and random selection of $k$ centroids.

2. Calculation of the distances of the respective objects to the centroids.

3. Assignment of the objects to the respective centroids according to the shortest distance.

4. Selection of centroids by calculation of the mean values of all assigned objects of a cluster.

5. Repetition of steps $2-4$ until there is no more change in the assignment of objects to clusters.

Figure 6 shows the allocation of an exemplary data set into four clusters.

For the investigations considered in this paper, the available data set is to be divided into subsets as shown in Fig. 6 and a representative data set of each cluster is to be used for training, as shown in Fig. 6c. The intent is to achieve a better distribution of the training data, an increase of the training

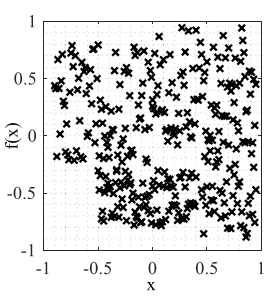

(a)

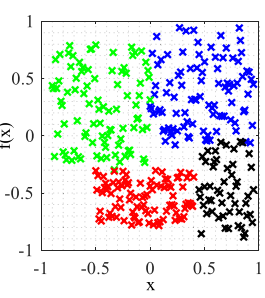

(b)

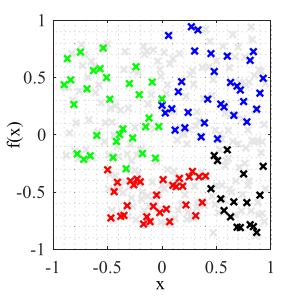

(c)
Fig. 6 Example representation of clustered data: a entire data set; $\mathbf{b}$ data set divided into four sub-clusters; $\mathbf{c}$ reduced data set 
speed, as well as a targeted tracking of possible failure cases of the neural network for post-training. In the following subsection, the generation of data sets is discussed and the network training is described.

\subsection{Data processing and network training}

For the approximation of the steering feel or hand torque, a feedforward network is used, which is comparable to [23], because one focus of this work is the automatic processing of the training data set and not the optimization of the network topology. The target value of the network is the torsion bar torque, which is detected by the standard torque sensor without additional sensors. The inputs of the network are analogous to [23] the steering wheel angle, the steering wheel angle speed, the lateral acceleration, the yaw rate, and the vehicle speed. In the reference vehicle, series production sensor technology is used as far as possible to record these variables. The goal is to be able to model the steering feel of any vehicle by means of similar neural networks with little effort by tapping the bus communication.

\subsubsection{Reference vehicle and maneuvers to design the training data set}

The data collection was carried out with a production C-segment vehicle. As the vehicle does not have an integrated steering wheel angle sensor, it was additionally equipped with a measurement steering wheel. Steering wheel angles and their speed were recorded via the measurement steering wheel and the remaining values via the series bus communication. For visualization of the measured data, a GPS unit was used, which is not relevant for network training. Since data preparation is carried out prior to the training by means of clustering, the sequence and the length or quantity of the operating ranges in the measurements have no decisive significance. The aim of the procedure is to ensure that all operating ranges are covered as well as possible, but without a fixed catalog of maneuvers. With the help of the clustering algorithm, underrepresented operating areas can be identified if necessary and the training data set can be expanded in a targeted manner. The data sets are the result of manually driven recordings. For validation of the trained network, a data set with the broadest possible spectrum of operating points is used. For network training, different maneuvers were performed. The reference vehicle was driven in different dynamic ranges during measurement runs. The data collection did not follow a fixed scheme, but was performed exclusively with the aim of subjectively exciting the vehicle in a wide range of possible states.

Figure 7 shows an exemplary measurement. On the basis of the shown trajectory, it becomes clear that in the context of manual driving, an attempt was made to record a large spread of different operating points. On the right-hand side, the spectrum of the recorded variables steering wheel angle, steering wheel angular velocity, yaw rate, lateral acceleration, and vehicle speed used as inputs for the network training is shown in form of box plots. It is noticeable that on one hand, a wide-operating range is shown, including speeds from 0 to about $120 \mathrm{~km} / \mathrm{h}$, steering angles of $300^{\circ}$, and lateral accelerations of $9.81 \mathrm{~m} / \mathrm{s}$. On the other hand, most of the maneuvers were performed at smaller steering angles, in the linear range and at speeds between 20 and $70 \mathrm{~km} / \mathrm{h}$. Measurements at the limit of the driving dynamic range, at very low speeds, or with very high steering angle speeds are the exception, but they play a central role in the exact representation of the steering torque. If the measurements are used for network training without further processing steps, it is possible that the marginal ranges are also underrepresented compared to the remaining driving maneuvers due to the small database and can therefore be poorly modeled.

\subsubsection{Division of the training data set into clusters}

The basic idea behind the use of the $k$-means method for the preparation of the training data set is to extract relevant data points from disordered, manual drives to accelerate network training and at the same time make it more efficient. Since, as described above, the main part of the scattered data lies in a certain driving dynamic range, a sufficient representation of marginal areas for the network training cannot be guaranteed. The clustering is multidimensional, over all input variables. Figure 8 illustrates the results for the data set described above.

On the right-hand side, the input variables and their distribution are normalized to a range from 0 to 1 . The black circles represent the data points, the colored filling corresponds to the assignment of a data point to a cluster. For the network training, the smallest cluster was used for an even distribution of the data points and the corresponding number of data points was randomly taken from each additional cluster. This guarantees an even distribution of data points over different driving ranges. Also, a large database for testing and validation is available, since all surplus data are
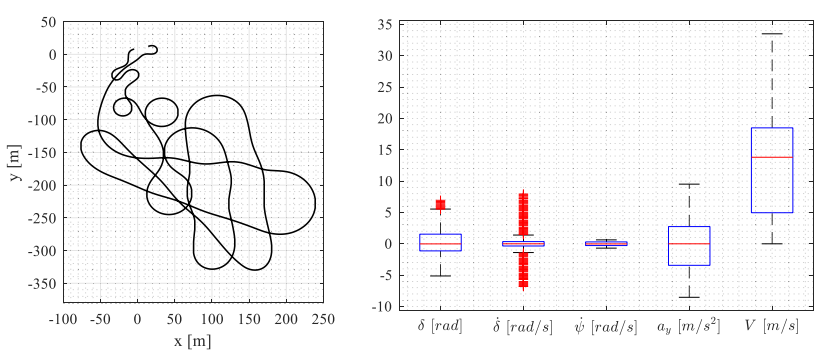

Fig. 7 Exemplary trajectory, distribution of network inputs, and target value to be approximated 


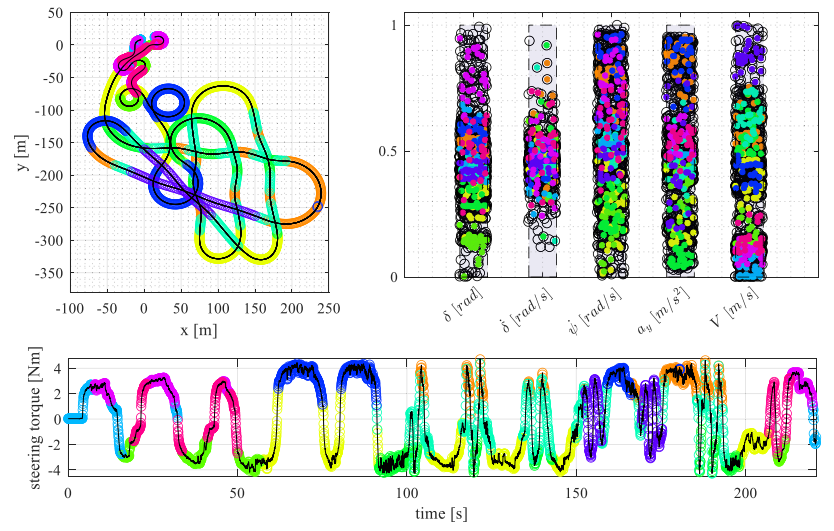

Fig. 8 Example of the division of the data set on the basis of an exemplary, manually driven trajectory

available for testing. If the network should fail in a certain cluster, or if the generalization properties should decrease, the training data set can be extended in a targeted manner. The trajectory divided into clusters shows that geometrically similar sections were assigned to equal clusters. Thus, curves with similar radii are assigned to the same cluster, although the section geometry is not a parameter, influencing the calculation of centroids and the clustering process in a direct manner. Also when looking at the steering torque, it becomes clear that similar sequences and amplitudes are divided into the same clusters, although the torque is not used for division into the clusters.

\subsection{Comparison of clustered and non-clustered training data sets}

In this section, the results of data set pre-processing using cluster methods will be compared to a non-processed data set before the different methods for steering torque modeling are compared. For the comparison, first, 50 feedforward networks each for three different cluster sizes and 50 networks with the not previously processed data set of manual drives were trained. The results for different synthetic maneuvers, which were not part of the training data, as well as the data of manual driving and the whole training data set are shown in Fig. 9.

The RMSE for all the trained networks is plotted over a normalized training time, where $100 \%$ represents the average training time for the 50 networks using all the available data for training. The networks were initialized with identical start weights, network architecture, and training parameters to ensure comparability between the networks. The figures illustrate that the training time is significantly reduced due to the more efficient design of the data set. Thus, for the differently clustered training data sets, an average reduction in training time of more than $50 \%$ can be achieved.
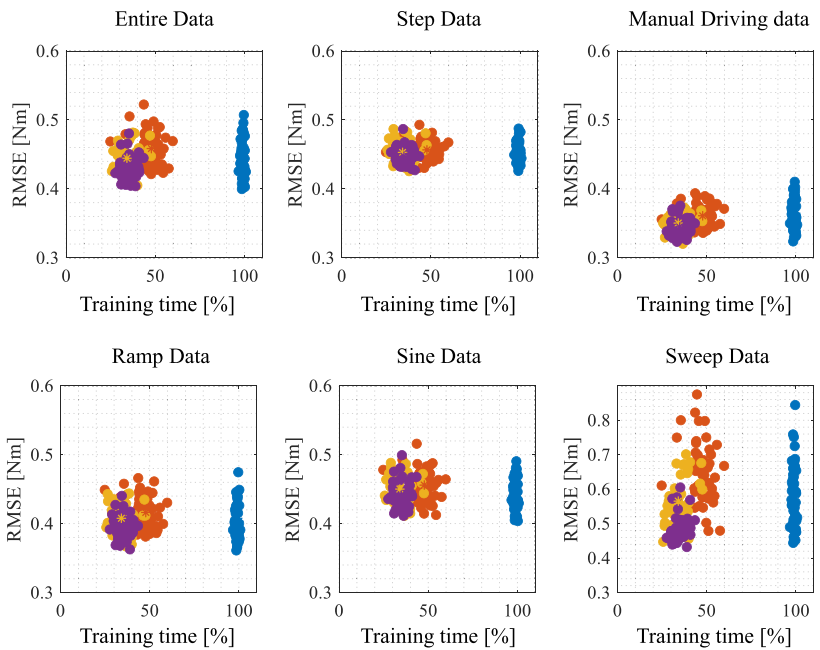

- no Cluster

- 10 Cluster

- 20 Cluster

- 40 Cluster

Fig. 9 Test errors for different maneuvers over normalized training time: differently clustered training data sets

Apart from the time saving, the aim of the presented method is to achieve a more homogeneous distribution of different driving scenarios in the training data set through efficient preprocessing of the training data. In addition, robustness in the application of the trained neural networks to unknown data and scenarios is to be increased by clustering the data set. To illustrate this, the RMSEs of the 50 networks of the respective configurations are plotted in Fig. 10, and shown for all considered maneuvers as well as the training, test, and validation data.

It becomes clear that, in particular, the training data sets, which were divided into a higher number of clusters, deliver significantly better results in terms of error spread than the networks that were trained with all available manual data points. In almost every maneuver (except the steering angle step), a lower spread of the error is generated by a data set divided into 20 clusters. In addition, in 40 clusters, an improvement of the RMSE can be observed in a large number of maneuvers, which is particularly evident in the test data. Only with a small number of clusters, no direct advantage in network performance can be detected. However, the results show that with a suitable number of clusters, a clear-added value for the training robustness as well as for the training time can be generated. In the future, methods for the optimized determination of the cluster number could further improve the efficiency of the method.

\section{Results}

In this section, the authors show the results of the two approaches to generate the steering feel. They are each compared to a measured steering torque of a series production 

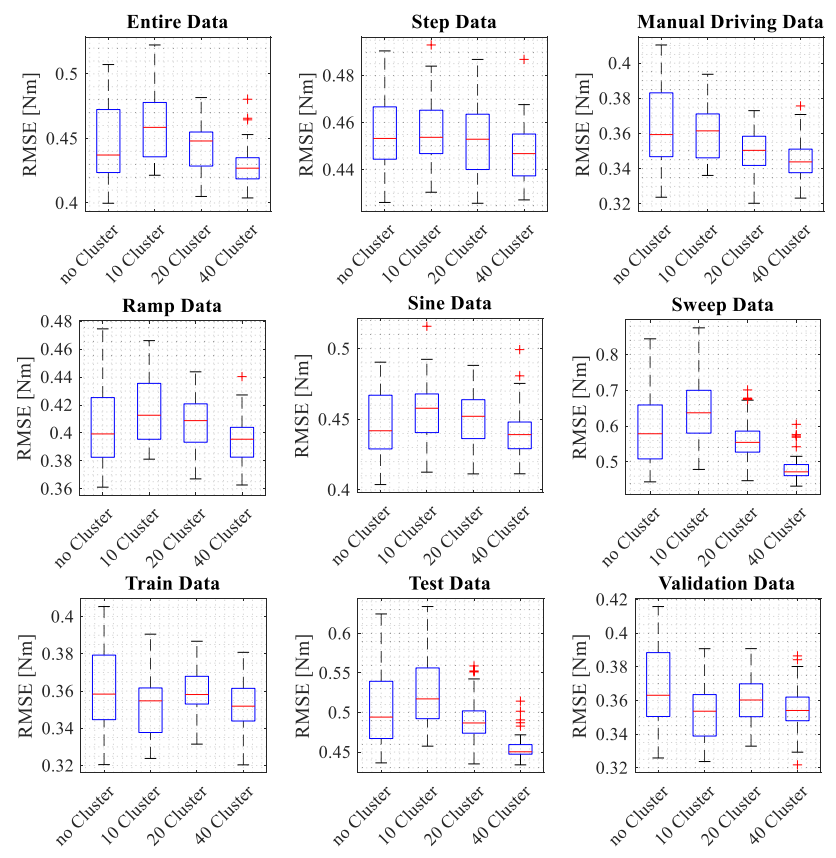

Fig. 10 RMSE for different maneuvers: differently clustered networks as well as a network trained with the entire data set

EPS system. The two very different approaches have their individual benefits and shortcomings that are highlighted in the following section. They are evaluated using three driving maneuvers. Two synthetic maneuvers have been performed using a steering robot and one measurement of a manual driving situation. The synthetic maneuvers consist of a damped steering step and a sine sweep from $0.1 \mathrm{~Hz}$ to $2 \mathrm{~Hz}$. Both have a maximum amplitude of $26^{\circ}$ which translates to a maximum lateral acceleration $a_{y}$ of $0.4 \mathrm{~m} / \mathrm{s}$. The manually driven maneuver shows a similar steering angle amplitude. The corresponding steering angles of each maneuver are displayed in the upper row of Fig. 11.

\subsection{Evaluation of the modular steering feel approach}

As described in Sect. 3, one approach for generating the steering feel is to modularly model the real-world phenomena and algorithms responsible for an EPS system's steering wheel torque. The parameters of the modular approach have been optimized to produce a steering feel that best resembles the one of the real vehicle. A comparison between the real-world data and the simulated steering wheel torque is illustrated in the third row of Fig. 11. Next to the measured and simulated steering wheel torques, the error between the two can be obtained for the three before mentioned driving maneuvers. There, it can be seen that the error stays below $0.5 \mathrm{Nm}$ for most of the time. The rare situations where it rises slightly above this threshold occur when the steering wheel either gets excited from a resting position or comes to a rest at a steering wheel angle $\varphi$ of around $0^{\circ}$. This becomes apparent at $2.2 \mathrm{~s}$ and $2.4 \mathrm{~s}$ in Fig. 11a. The effect can be explained by the deviation between the friction model and the complex real-world friction effects in the measurement. The deviation is amplified as the friction model is used twice—once for modeling the feedback actuator's friction and once for generation the friction part of the modular steering feel. Figure 11c shows another type of deviation. Specifically in the long left hand turn from 80 to $110 \mathrm{~s}$, the modular approach exhibits a constant error of approximately $0.3 \mathrm{Nm}$. This behavior is a result of the velocity dependency of the approach and the fact that it has only been adjusted to $100 \mathrm{~km} / \mathrm{h}$. Therefore, diverging velocities result in a diverging torque level. However, as the error mainly stays below $10 \%$ of the maximum amplitude and always below the threshold of $0.8 \mathrm{Nm}$ as defined by [4], this approach is suitable to adequately model an EPS system's steering torque.

\subsection{Evaluation of the ANN approach}

As depicted in Fig. 11, the neural network performs best in the steering ramp maneuver (a) and the manual maneuver (c) The error remains below $0.8 \mathrm{Nm}$. However, the ANN approach struggles to capture the increased steering wheel frequency of the sinusoidal sweep maneuver (b) resulting in higher errors.

In the synthetic maneuvers (a) and (b), it can be seen that there is a larger error during the initial excitation of the steering wheel from rest. The initial error is due to the behavior of a steering wheel when held in a straight position by the steering robot rather than being at rest at $0 \mathrm{Nm}$. This offset is presumably due to a slight offset in the steering robot's center position calibration. The result is that the steering robot produces a torque at rest to keep the steering wheel from swinging back to a rest position based on the wheel's current position. A similar behavior can be seen at the end of the first maneuver in (a), wherein the steering wheel returns to position of $0^{\circ}$ and while the neural network results in $0 \mathrm{Nm}$ torque, preventing the steering wheel from turning into a negative direction, the steering robot holds the steering wheel at 0 with a negative torque.

The sinusoidal maneuver seen in Fig. $11 \mathrm{~b}$ proved to be difficult for the ANN approach. The discrepancy and larger errors seen in the maneuver can be attributed to the lack of information presented to the neural network during training for higher steering frequencies as well as a slight delay that became apparent in training the networks. While the amplitudes of the ANN approach are similar to those produced by the EPS Steering torque, the phase delay by the neural network caused increasing discrepancies. While the frequency increases, the delay between the neural network and the EPS Steering torque 

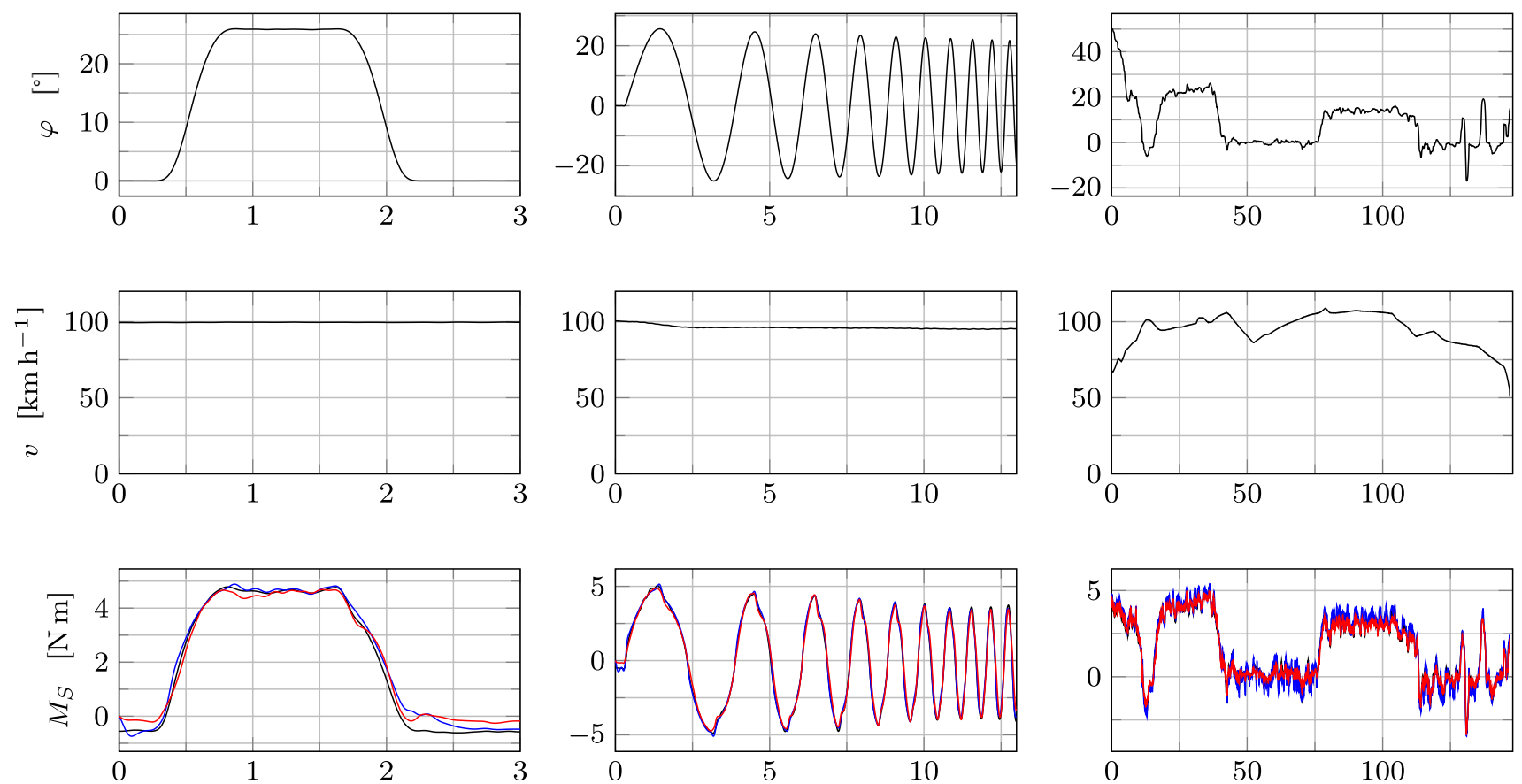

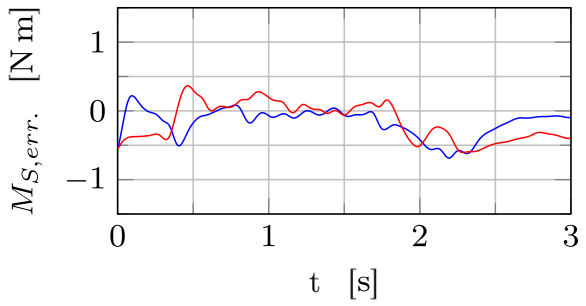

(a)

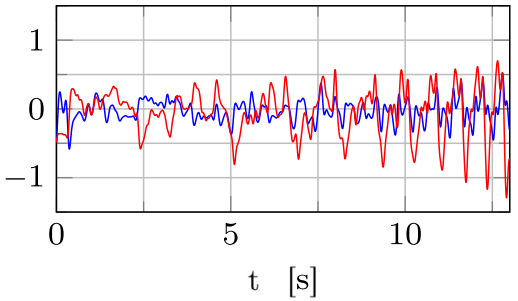

(b)

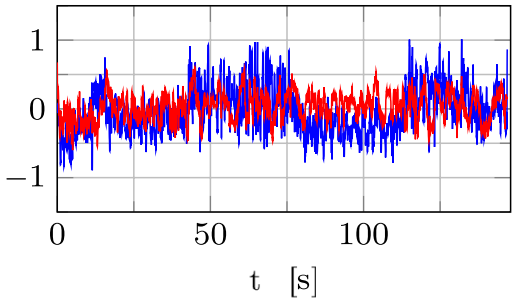

(c)

Measurement — Modular — Neural Network

Fig. 11 Comparison of target and modeled steering torques in maneuvers: a damped step, $\mathbf{b}$ sweep, and $\mathbf{c}$ manual steering

becomes more pronounced. Furthermore, the neural network is trained using five inputs rather than merely the steering angle and velocity. During the sinusoidal maneuver, the steering angle frequency is increased to create the sinusoidal sweeps resulting in higher steering wheel velocities but decreasing yaw rates and lateral accelerations. From the results, it becomes apparent that the neural network places more emphasis on the yaw rate and lateral acceleration. As a result, an increasing steering frequency with decreasing yaw rate and lateral acceleration generates a decreasing or constant resulting torque, while the real EPS-torque increases or remains constant. Additional training data including sweep maneuvers could improve the performance of the neural network, but was not further explored here.

\subsection{Comparison of the two approaches}

As mentioned before, the two different approaches each have their individual advantages and shortcomings. The overall performance of each approach in the chosen maneuvers displayed in Fig. 11 shall be characterized by the RMSE of the steering torque. The respective values can be obtained from Table 1.

For maneuver (a), the performance of the two approaches is similar, differing less than $20 \%$. Moreover, the existing deviation can largely be attributed to the fact that the measurement's torque at $0^{\circ}$ is not equal to $0 \mathrm{Nm}$. In maneuver (b), it can be seen that the ANN approach fits the measurement data well in terms of amplitude, but has an increasing delay as the frequency rises. The modular approach appears 
Table 1 RMSE of the different approaches and maneuvers

\begin{tabular}{llll}
\hline Approach & Damped step (a) & $\begin{array}{l}\text { Sinusoidal } \\
\text { sweep (b) }\end{array}$ & $\begin{array}{l}\text { Manual } \\
\text { driving } \\
\text { (c) }\end{array}$ \\
\hline Modular & 0.264 & 0.159 & 0.327 \\
Neural network & 0.313 & 0.342 & 0.201 \\
\hline
\end{tabular}

to cope better with higher frequencies of up to $2 \mathrm{~Hz}$. That results in the modular approach's RMSE being 53\% lower than the neural network's.

In maneuver (c), which has been performed at a varying velocity the ANN approach performs better throughout. This is rewarded with a RMSE of 0.201 as opposed to the modular approach's 0.327 . This result can be explained by the adjustment to differing velocities of the two approaches. While the neural network has been trained using a wide range of velocities, the modular approach has only been tuned to fit the EPS system's torque at $100 \mathrm{~km} / \mathrm{h}$.

All in all, it can be stated that both approaches can be used to adequately model an EPS system's steering feel on a steer-by-wire system. The modular approach relies on individual applications for certain velocity ranges, which requires more tuning effort. That cost however comes with the benefit of increased tunability, which is needed later to finally adjust the steering feel after simulation on the hardware. The neural network is not tunable per se, but its performance very much relies on the maneuvers used for training.

\section{Conclusion}

In the present study, two different approaches for modeling the steering feel of a steer-by-wire system have been examined. The target was to produce a steering torque that closely resembles that of an EPS system in defined maneuvers. The first approach is a modular one that was designed to model an EPS system's functions and behavior. The other is based on an ANN.

As a first step, a simulation model of an actual steer-bywire system was created. It consists of the rack actuator and its position controller and the steering wheel actuator with its torque controller. Both are embedded in a vehicle model that accurately represents a C-segment car. After validation of the simulated torque through a comparison with measurement data, the two steering feel approaches are described. The individual modules of the first approach are presented and their optimization described. For the ANN approach, not only the ANN itself and the training are subject of the study but also a novel method of clustering the training data. This new methods allows to cut training time in half.
The research shows that both approaches are suitable for representing an EPS system's steering feel on a steerby-wire system. Each approach has its own advantages and drawbacks. The modular approach shows its strength in the velocity range for which it has been optimized, especially at higher frequencies. For manual maneuvers and outside its velocity range, the deviation becomes larger. However, it also has the advantage of possible manual tunability for in-vehicle applications. Tuning the approach for a wider speed range is advisable for future work to address the drawbacks described. The neural network shows good results for manual driving with varying speed and varying steering inputs, since these driving maneuvers are sufficiently well represented in the training data. For the synthetic maneuvers, it is shown that high-frequency changes of the steering wheel angle are not sufficiently included in the training database. Accordingly, the accuracy of the neural network decreases with increasing frequency. To improve the results, an extension of the data set with synthetic maneuvers is useful. With the help of the presented clustering approach, a uniform distribution of the training data can be provided, which adds value to the network training and specifically addresses white spots in the training data set. To combine the advantages of both approaches and to eliminate as many disadvantages as possible, a combination of both approaches is suggested for further research. This way, a basic steering feel can be represented by mathematical functions and nonlinear influences can be integrated into the concept by an ANN module. The modular, tunable approach would mainly be responsible for the steering feel, while the ANN module can be used to compensate for unwanted effects such as excess friction or even to model a useful amount of friction.

Funding Open Access funding enabled and organized by Projekt DEAL.

\section{Declarations}

Conflict of interests On behalf of all authors, the corresponding author states that there is no conflict of interest.

Open Access This article is licensed under a Creative Commons Attribution 4.0 International License, which permits use, sharing, adaptation, distribution and reproduction in any medium or format, as long as you give appropriate credit to the original author(s) and the source, provide a link to the Creative Commons licence, and indicate if changes were made. The images or other third party material in this article are included in the article's Creative Commons licence, unless indicated otherwise in a credit line to the material. If material is not included in the article's Creative Commons licence and your intended use is not permitted by statutory regulation or exceeds the permitted use, you will need to obtain permission directly from the copyright holder. To view a copy of this licence, visit http://creativecommons.org/licenses/by/4.0/. 


\section{References}

1. Balachandran, A., Gerdes, J.C.: Artificial steering feel design for steer-by-wire vehicles. IFAC Proc. Vol. 46, 404-409 (2013)

2. Bellman, R.: An Introduction to Artificial Intelligence: Can Computers Think? Boyd and Fraser, San Francisco (1978)

3. Böhm, F.: Zur Mechanik des Luftreifens. Dissertation. Technische Universität Stuttgart, Stuttgart (1966)

4. Buschardt, B.: Synthetische Lenkmomente. Dissertation. Technische Universität Berlin, Berlin (2003)

5. Celebi, M.E., Kingravi, H.A., Vela, P.A.: A comparative study of efficient initialization methods for the k-means clustering algorithm. Expert Syst. Appl. 40, 200-210 (2013)

6. Fankem, S.: Steering feel generation in steer-by-wire vehiclesmodular steering torque computation and requirements for the hand wheel actuator. In: 5th International Munich Chassis Symposium, pp. 471-528 (2014)

7. Glorot, X., Bengio, Y.: Understanding the difficulty of training deep feedforward neural networks. In: Proceedings of the International Conference on Artificial Intelligence and Statistics, pp. 249-256 (2010)

8. Koch, T.: Untersuchungen zum Lenkgefühl von Steer-by-Wire Lenksystemen. Dissertation. Technische Universität München (2010)

9. Krüger, T.: Zur Anwendung neuronaler Netzwerke in adaptiven Flugregelungssystemen. Dissertation. Technische Universität Carolo-Wilhelmina zu Braunschweig (2012)

10. Krupka, P., Lukowicz, P., Kreis, C., Boßdorf-Zimmer, B.: Learned steering feel by a neural network for a steer-by-wire system. In: 10th International Munich Chassis Symposium, pp. 449-464 (2019)

11. LeCun, Y., Bengio, Y., Hinton, G.: Deep learning. Nature 521, 436-444 (2015)

12. Likas, A., Vlassis, N., Verbeek, J.: The global K-means clustering algorithm. Pattern Recognit. 36, 451-461 (2002)

13. Na, S., Xumin, L., Yong, G.: Research on k-means clustering algorithm: an improved k-means clustering algorithm. In: Third International Symposium on Intelligent Information Technology and Security Informatics, pp. 63-67 (2010)
14. Nair, V., Hinton, G.: Rectified linear units improve restricted Boltzmann machines. In: Proceedings of the 27th International Conference on International Conference on Machine Learning, pp. 807-814 (2010)

15. Nippold, C.: Prüfstandsbasierte Lenkungsoptimierung im Mittenbereich. Dissertation. Institut für Fahrzeugtechnik, Technische Universität Braunschweig, Braunschweig (2017)

16. Rich, E., Knight, K.: Artificial Intelligence, 2nd edn. McGrawHill, New York (1991)

17. Riekert, P., Schunck, T. E.: Zur Fahrmechanik des gummibereiften Kraftfahrzeuges. In: Ingenieur Archiv Bd. 11 1940, S. $210 \mathrm{ff}$

18. Russell, S., Norvig, P.: Artificial Intelligence: A Modern Approach, 3rd edn. Series in Artificial Intelligence, Chapter 1. Prentice Hall, Upper Saddle River (2010)

19. Schnetter, P.: Sliding-Mode-Lernverfahren für neuronale Netzwerke in adaptiven Regelungssystemen. Dissertation. Technische Universität Carolo-Wilhelmina zu Braunschweig (2017)

20. Sculley, D.: Web-scale K-means clustering. In: Proceedings of the 19th International Conference on World Wide Web, 1177-1178, (2010)

21. Steinley, D.: K-means clustering: a half-century synthesis. Br. J. Math. Stat. Psychol. 59, 1-34 (2006)

22. Talukdar, S., Adeel Awa, M., Tremlett, A., Sastry, V., Purdy, D.: Preview Based Vehicle Steering Control using Neural Networks. SAE International, Wareendale (2013)

23. Van Ende, K.T.R., Schaare, D., Kaste, J., Kücükay, F., Henze, R., Kallmeyer, F.K.: Practicability study on the suitability of artificial, neural networks for the approximation of unknown steering torques. Veh. Syst. Dyn. 54, 1362-1383 (2016)

24. Winde, M.: Systematische Bewertung und Ertüchtigung von industriellen Regelkreisen in verfahrenstechnischen Komplexen. Dissertation. Ruhruniversität Bochum, Bochum (2009)

25. Zalik, K.R.: An efficient k'-means clustering algorithm. Pattern Recognit. Lett. 29(9), 1385-1391 (2008)

Publisher's Note Springer Nature remains neutral with regard to jurisdictional claims in published maps and institutional affiliations. 